\title{
The effect of icotinib combined with chemotherapy in untreated non-small-cell lung cancer that harbored EGFR-sensitive mutations in a real-life setting: a retrospective analysis
}

This article was published in the following Dove Press journal:

OncoTargets and Therapy

\section{Lulu Wang \\ Yan Li \\ Luchun $\mathrm{Li}$ \\ Zhijuan Wu \\ Dan Yang \\ Huiwen $\mathrm{Ma}$ \\ Donglin Wang}

Oncology Department, Chongqing

University Cancer Hospital \&

Chongqing Cancer Institute \&

Chongqing Cancer Hospital, Shapingba

District, Chongqing, China
Correspondence: Huiwen Ma;

Donglin Wang

Chongqing University Cancer

Hospital \& Chongqing Cancer

Institute \& Chongqing Cancer Hospital,

18I Hanyu Road, Shapingba District,

Chongqing 400000, China

Email851487I03@qq.com;

donglinw@2Icn.com
Purpose: This study was conducted to compare the efficacy of a combination of icotinib and chemotherapy with icotinib or chemotherapy alone in untreated non-small cell lung cancer (NSCLC) patients harboring epidermal growth factor receptor (EGFR)-sensitive mutations and to analyze the curative effect of different treatments on different genetic mutations (EGFR 19 exon deletion and L858R mutation) in a real-life setting.

Patients and methods: One hundred ninety-one patients were studied in this retrospective analysis from January 2013 to December 2015. The baseline characteristics, curative effects and adverse events of patients were analyzed. The primary endpoint was progression free survival (PFS).

Results: Longer PFS and overall survival (OS), and better objective response rate (ORR) were observed in the combination group compared to icotinib or chemotherapy along. For patients with an EGFR 19 exon deletion, the PFS, OS, and ORR in the combination group were superior to those in the icotinib or chemotherapy group. For the patients with the EGFR L858R mutation, better PFS and ORR were observed in the combination group, but OS was not obviously prolonged. Grade 3 or 4 adverse events were most commonly reported with combination therapy or chemotherapy alone. No possible drug-related interstitial lung disease or of drug related deaths occurred.

Conclusion: The combination of icotinib and chemotherapy in patients with untreated NSCLC harboring sensitive EGFR mutations resulted in improved PFS and OS, especially in those who harbored the EGFR exon 19 deletion.

Keywords: non-small-cell lung cancer, EGFR-TKI, icotinib, chemotherapy, first-line treatment

\section{Introduction}

Lung cancer is the leading cause of cancer mortality worldwide, with $\sim 80 \%-85 \%$ patients suffering from non-small-cell lung cancer (NSCLC). ${ }^{1}$ The majority of patients with NSCLC have advanced stage (IIIB or IV) disease at the time of diagnosis and thus are not candidates for surgery. For these patients, systemic chemotherapy remains the standard treatment option, but its effects are limited and its severe adverse side effects significantly affect patient's quality of life. ${ }^{2}$ The epidermal growth factor receptor (EGFR)-dependent pathway plays an important role in NSCLC proliferation; it is activated in more than half of patients with NSCLC. ${ }^{3}$ Small-molecule EGFR-tyrosine kinase inhibitors (EGFR-TKIs) can block the EGFR-dependent pathway; their development provides a new treatment option and offers new hope for patients with advanced NSCLC. Certain NSCLC patient subgroups (ie, women, East Asians, never-smokers, and adenocarcinoma) more commonly exhibit EGFR-sensitive mutations (deletion of 
exon 19 and the L858R mutation in exon 21). ${ }^{4,5}$ Patients with EGFR-mutant NSCLC are sensitive to EGFR-TKIs, such as gefitinib, erlotinib, and icotinib, with the response to EGFRTKIs reaching $70 \%-80 \%$. The median survival time has been reported to reach $20-30$ months, with a significant improvement in patient quality of life for those receiving EGFR-TKIs compared to those on chemotherapy. ${ }^{6,7}$ Thus, EGFR-TKIs have been recommended in the National Comprehensive Cancer Network guidelines as a first-line treatment option and are commonly used for patients with advanced NSCLC who harbor EGFR-sensitive mutations.

Despite its high remission and cure rates, TKI treatment will inevitably lead to acquired drug resistance. ${ }^{8}$ Various resistance mechanisms have been reported. The acquired T790M mutation in exon 20 of EGFR is the most common mechanism of resistance to first-generation EGFR-TKI and is present in $\sim 50 \%-60 \%$ of resistant cases..$^{9-11}$ Third-generation EGFR inhibitors, such as AZD9291 (osimertinib, mereletinib) and CO-1686 (rociletinib), have emerged as potential agents to block the growth of EGFR T790M-positive tumors. ${ }^{12,13}$ Recently, in an effort to delay the development of drug resistance and improve the curative effect of EGFRTKI as a first-line treatment, close attention has been paid to the combination of EGFR-TKI and chemotherapy as firstline treatment for EGFR-mutant advanced NSCLC. Given their different mechanisms of action, the combination of EGFR-TKI and chemotherapy may improve outcomes. ${ }^{14}$ However, the results of several previous randomized controlled trials, including INTACT-1, INTACT-2, TRIBUTE, and TALENT, showed that the combination was no more beneficial than chemotherapy alone. ${ }^{15-18}$ The possible reason for the failure to achieve positive results was not selecting patients harboring EGFR-sensitizing mutations. The studies carried out by Fred R evaluated the treatment outcomes with erlotinib alone or intercalating erlotinib and chemotherapy in advanced NSCLC patients who did not receive any prior or current anticancer therapy. The results showed that patients with activated EGFR mutations treated with erlotinib alone had better response rates, better progression-free survival (PFS), and better overall survival (OS) than those who received intercalating therapy. ${ }^{19}$ The study of CALGB 30406 performed a similar comparison and suggested that improving the PFS of patients with EGFR-mutant NSCLC by adding chemotherapy to erlotinib is unlikely. ${ }^{20}$ However, in the studies of B Han and Ying Cheng, the combination of gefitinib and chemotherapy improved PFS compared with gefitinib alone in patients with advanced NSCLC and activated EGFR mutations. ${ }^{21,22}$ The efficacy of first-line treatment with a combination of EGFR-TKI and chemotherapy in improving survival remains controversial.

Icotinib (Betta Pharmaceuticals Co., Ltd, Hangzhou, China), a first-generation EGFR-TKI, is an orally administered, reversible small-molecule EGFR-TKI, which has independent intellectual property rights in the People's Republic of China. Icotinib has shown equivalent curative effects, milder adverse reactions, better tolerability, and lower prices compared with gefitinib and erlotinib. ${ }^{23}$ Its most common adverse events include rash and diarrhea, and no cases of interstitial lung disease have been reported. Icotinib is an important tool in the People's Republic of China for the first-line treatment of patients with advanced NSCLC who harbor sensitive EGFR mutations. In previous studies, most combination therapy focused on gefitinib or erlotinib, so the curative effect and safety of icotinib combined with chemotherapy remain unclear. To improve the effects of icotinib as the first-line treatment and to provide a clinical rationale for the use of a combination of EGFR-TKI and chemotherapy, we performed this retrospective analysis to comprehensively examine the overall efficacy and safety of icotinib in combination with chemotherapy as the first-line treatment of advanced NSCLC in patients harboring EGFR mutations in an uncontrolled real-life setting. In addition, several studies have reported that patients with advanced NSCLC and an EGFR exon19 deletion may have a longer PFS or OS following treatment with EGFR-TKI compared to patients with the L858R mutation. ${ }^{24,25}$ Therefore, in our study, we also analyzed the curative effect of different treatments on different genetic mutations (EGFR exon 19 deletion or L858R mutation).

\section{Patients and methods Patients}

Between January 2013 and December 2015, a total of 191 patients who met the inclusion criteria were eligible for our retrospective analysis. The patients' records were anonymized and de-identified prior to analysis. This study was approved by Chongqing Cancer Hospital Ethics Committee. Patients in our study have signed written informed consent.

The main inclusion criteria were 1 ) age $\geq 18$ years; 2) Eastern Cooperative Oncology Group performance status (ECOG PS) $\leq 2 ; 3$ ) pathologically diagnosed advanced NSCLC (stages IIIB or IV); 4) NSCLC harboring activated EGFR mutation (primarily exon 21 L858R point mutation or exon 19 deletion); 5) radiologically measurable or evaluable disease; 6) no other cancer; and 7) first-line treatment with received icotinib (125 mg tid)+chemotherapy or icotinib alone (125 mg tid) or chemotherapy alone. The main 
exclusion criterion was if patients had received any prior or concurrent anticancer therapy for advanced NSCLC. Previous treatment during earlier-stage NSCLC was permitted.

Data collected from the medical records included age, sex, smoking history, EGFR mutation type, best response, toxicities, and survival data.

\section{Assessment of efficacy and adverse events}

The Response Evaluation Criteria in Solid Tumors was used to evaluate tumor response. The primary end point was PFS, which was defined as the period from the initial administration of icotinib or chemotherapy to tumor progression, death from any cause, or the last follow-up (calculated according to the event that occurred first). Secondary end points included OS, objective response rate (ORR), disease control rate (DCR), and toxicities. OS was defined as the span between the initiation of icotinib or chemotherapy and the date of death or the deadline of follow-up. ORR was defined as the rate of complete response (CR) and partial response (PR). DCR was defined as the rate of CR, PR, or stable disease. Adverse events were assessed according to Common Terminology Criteria for Adverse Events of the National Cancer Institute (version 3.0).

\section{Statistical analysis}

The chi-squared $\left(\chi^{2}\right)$ test was used for comparisons of ORR and DCR intergroups at a significance level of 5\% $(a=0.05$, two-sided). The Kaplan-Meier method was utilized to obtain PFS and OS. The log-rank test was used to compare the significance between groups. The multivariate Cox regression model was used to calculate the hazard ratios. $p$-values of $<0.05(p<0.05)$ were considered statistically significant. SPSS software (version 22.0; SPSS Inc., Chicago, IL, USA) was used for all statistical analyses.

\section{Results}

\section{The overall curative effect and safety} evaluation of different treatments

\section{Patient characteristics}

The study population included 92 men (48.2\%) and 99 women $(51.8 \%)$. The median age of the population was 60.8 years (range, 39-80 years). Of the 191 patients, 59 received icotinib+chemotherapy (group A), 75 received icotinib alone (group B), and 57 received chemotherapy alone (group C). The patients' baseline characteristics (age, sex, smoking history, ECOG PS, and EGFR mutation) are summarized in Table 1. The three groups were comparable with respect to disease and demographic characteristics. All patients had histologically proven adenocarcinoma of the lung. Most patients were nonsmokers with good ECOG PS (0-1). Approximately $53.4 \%$ of the patients $(n=102)$ were found to have an EGFR exon 19 deletion, while 46.6\% $(n=89)$ had an EGFR exon $21 \mathrm{~L} 858 \mathrm{R}$ point mutation in the EGFR gene. In group A, the chemotherapy regimens contained pemetrexed+cisplatin/ carboplatin $(n=20,33.9 \%)$ and paclitaxel+cisplatin/ carboplatin $(n=39,66.1 \%)$. In group $C$, the chemotherapy regimens contained pemetrexed+cisplatin/carboplatin $(n=25$, $43.9 \%)$ and paclitaxel+cisplatin/carboplatin $(n=32,56.1 \%)$.

\section{Short-term curative effects}

As shown in Table 2, the ORR was higher in group A than in group B $(64.4 \%$ vs $46.7 \%, p<0.05)$. ORR in group B was

Table I Characteristics of all patients

\begin{tabular}{|c|c|c|c|c|}
\hline Characteristics & I+C (group A, n=59) & I (group B, n=75) & $C$ (group C, $n=57$ ) & Total $(n=191)$ \\
\hline Median age (years) & 62.0 & 60.6 & 59.9 & 60.8 \\
\hline \multicolumn{5}{|l|}{ Sex } \\
\hline Male & $40.7 \%(n=24)$ & $37.3 \%(n=28)$ & $47.4 \%(n=27)$ & $41.4 \%(n=79)$ \\
\hline Female & $59.3 \%(n=35)$ & $62.7 \%(n=47)$ & $52.6 \%(n=30)$ & $58.6 \%(n=\mid 12)$ \\
\hline \multicolumn{5}{|l|}{ Smoking history } \\
\hline Nonsmoker & $61.0 \%(n=36)$ & $65.3 \%(n=49)$ & $59.6 \%(n=34)$ & $62.3 \%(n=119)$ \\
\hline Smoker & $39.0 \%(n=23)$ & $34.7 \%(n=26)$ & $40.4 \%(n=23)$ & $37.7 \%(n=72)$ \\
\hline \multicolumn{5}{|c|}{ ECOG performance status score } \\
\hline $0-1$ & $100 \%(n=59)$ & $96.0 \%(n=72)$ & $98.2 \%(n=56)$ & $97.9 \%(n=187)$ \\
\hline 2 & $0 \%(n=0)$ & $4.0 \%(n=3)$ & $1.8 \%(n=1)$ & $2.1 \%(n=4)$ \\
\hline \multicolumn{5}{|c|}{ Type of EGFR mutation } \\
\hline Exon 19 deletion & $52.5 \%(n=31)$ & $56.0 \%(n=42)$ & $50.9 \%(n=29)$ & $53.4 \%(n=102)$ \\
\hline L858R & $47.5 \%(n=28)$ & $44.0 \%(n=33)$ & $49.1 \%(n=28)$ & $46.6 \%(n=89)$ \\
\hline \multicolumn{5}{|l|}{ Histologic diagnosis } \\
\hline Adenocarcinoma & $100 \%$ & $100 \%$ & $100 \%$ & $100 \%$ \\
\hline
\end{tabular}

Abbreviations: C, chemotherapy; ECOG, Eastern Cooperative Oncology Group; EGFR, epidermal growth factor receptor; I, icotinib; I+C, icotinib+chemotherapy. 
Table 2 Short-term curative effects of all patients

\begin{tabular}{llll}
\hline $\begin{array}{l}\text { Curative } \\
\text { effects }\end{array}$ & $\begin{array}{l}\text { I+C (group A, } \\
\mathbf{n}=\mathbf{5 9})\end{array}$ & $\begin{array}{l}\text { I (group B, } \\
\mathbf{n}=\mathbf{7 5})\end{array}$ & $\begin{array}{l}\mathbf{C} \text { (group C, } \\
\mathbf{n}=\mathbf{5 7})\end{array}$ \\
\hline $\begin{array}{l}\text { Best response } \\
\text { CR }\end{array}$ & $\begin{array}{l}\text { I.7\% }(\mathrm{n}=1) \\
\text { PR }\end{array}$ & $0 \%(\mathrm{n}=0)$ & $0 \%(\mathrm{n}=0)$ \\
SD & $62.7 \%(\mathrm{n}=37)$ & $46.7 \%(\mathrm{n}=35)$ & $26.3 \%(\mathrm{n}=15)$ \\
PD & $30.5 \%(\mathrm{n}=18)$ & $45.3 \%(\mathrm{n}=34)$ & $43.9 \%(\mathrm{n}=25)$ \\
ORR $(\%)$ & $5.1 \%(\mathrm{n}=3)$ & $8.0 \%(\mathrm{n}=6)$ & $29.8 \%(\mathrm{n}=17)$ \\
DCR $(\%)$ & 64.4 & 46.7 & 26.3 \\
\hline
\end{tabular}

Abbreviations: C, chemotherapy; CR, complete remission; DCR, disease control rate; I, icotinib; I+C, icotinib+chemotherapy; ORR, overall response rate; PD, progression disease; PR, partial disease; SD, stable disease.

better than that in group $\mathrm{C}(46.7 \%$ vs $26.3 \%, p<0.05)$. The DCRs in group A, group B, and group C were 94.9\%, 92.0\%, and $70.2 \%$, respectively; there was no statistically significant difference between group A and group B $(p>0.05)$, both of which were superior to group $\mathrm{C}(p<0.05)$.

\section{Survival analysis}

As of January 2017, 188 patients (98.4\%) had reached the end point of disease progression or death. The median progressionfree survival (mPFS) for the patients in group A, group B, and group C was 11.569 months (95\% CI, 10.347-12.764), 8.607 months (95\% CI, 7.806-9.407), and 6.544 months (95\% CI, 5.634-7.453), respectively. The results suggest a favorable trend in PFS for the patients treated with icotinib+chemotherapy compared to those treated with icotinib alone $(p<0.001$, Figure 1A). In addition, patients treated with icotinib alone had significantly longer PFS compared to those who were treated with chemotherapy ( $p<0.05$, Figure $1 \mathrm{~A})$. At the last follow-up, $38.7 \%(n=74)$ patients were still alive (24 patients in group $A$; 31 patients in group B; 19 patients in group C). The median

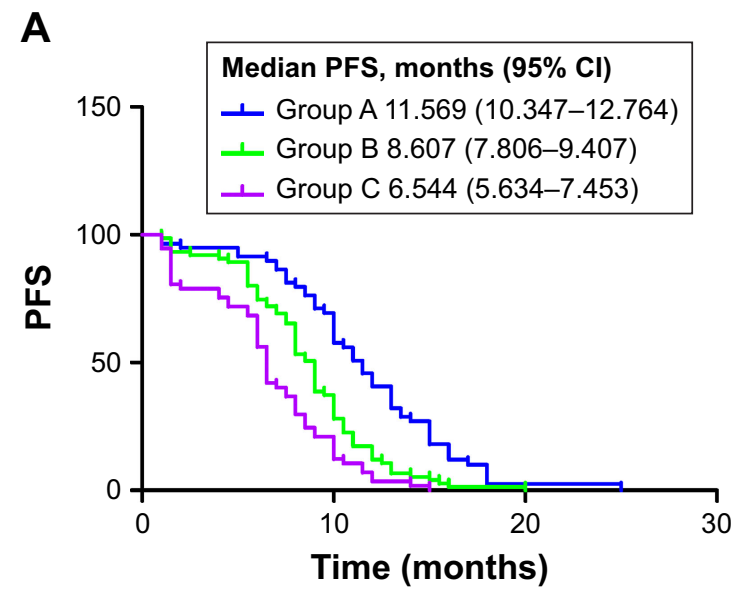

OS was 24.379 months (95\% CI, 21.125-27.632) in group A, 20.244 months $(95 \% \mathrm{CI}, 18.493-21.995)$ in group $\mathrm{B}$, and 20.633 months $(95 \%$ CI, 18.148-23.117) in group C. While the OS data are immature, better OS was observed in group A compared to group $\mathrm{B}(p<0.05$, Figure $1 \mathrm{~B})$. The OS comparison between group $\mathrm{B}$ and group $\mathrm{C}$ did not reach statistical significance $(p>0.05$, Figure 1B).

\section{Adverse events}

As shown in Table 3, The most common adverse events in group A were hematologic toxicities (neutropenia 50.8\%; anemia $40.7 \%$; thrombocytopenia $18.6 \%$; leukopenia $22.0 \%$ ), gastrointestinal reactions (nausea $39.0 \%$; vomiting $39.0 \%$; diarrhea $33.9 \%$; constipation $11.9 \%$ ), skin reactions (rash 52.5\%), and liver function impairment (aspartate transaminase $[\mathrm{AST}] /$ alanine transaminase [ALT] elevation $37.3 \%)$. Other common adverse events included stomatitis $(22.0 \%)$, fatigue $(25.4 \%)$, and alopecia $(16.9 \%)$. The most common adverse events in group B were rash $(30.7 \%)$, diarrhea (21.3\%), and AST/ALT elevation (12.0\%). The most common adverse events in group $\mathrm{C}$ were hematologic toxicities (neutropenia 42.1\%; anemia 50.9\%; thrombocytopenia $22.8 \%$; leukopenia $22.8 \%$ ), gastrointestinal reaction (nausea $40.4 \%$; vomiting $40.4 \%$; diarrhea $21.0 \%$; constipation $21.0 \%$ ), skin reactions (rash 14.0\%), and liver function impairment (AST/ALT elevation 24.6\%). Other common adverse events included stomatitis (5.3\%), fatigue (24.6\%), and alopecia (22.8\%). Hematologic adverse events and gastrointestinal reactions occurred with similar incidence in groups $\mathrm{A}$ and $\mathrm{C}$, consistent with the toxicity profile of chemotherapy. Icotinib did not seem to exacerbate these toxicities. The adverse events related to icotinib were skin

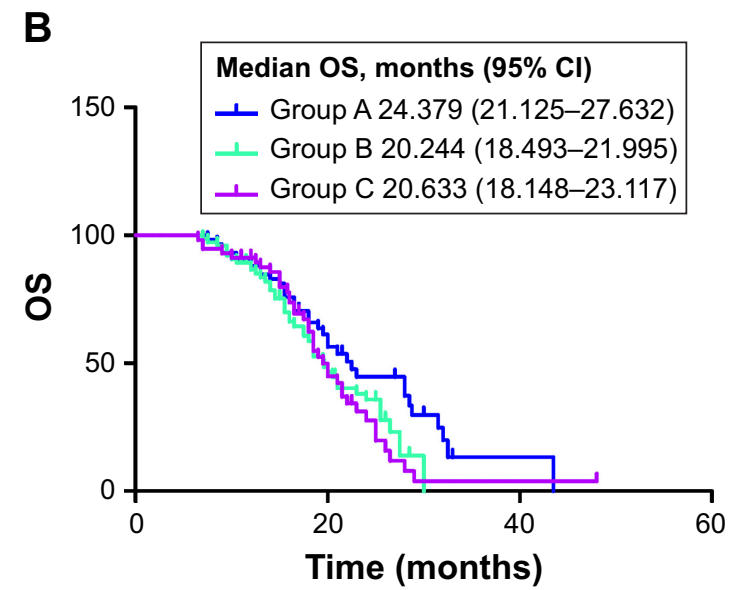

Figure I Kaplan-Meier curve of PFS (A) and OS (B) for all patients.

Notes: Group A, 59 patients received icotinib+chemotherapy; Group B, 75 patients received icotinib alone; Group C, 57 patients received chemotherapy alone. Abbreviations: OS, overall survival; PFS, progression-free survival. 
Table 3 Adverse events

\begin{tabular}{|c|c|c|c|c|c|c|}
\hline \multirow[t]{2}{*}{ Toxicity } & \multicolumn{2}{|c|}{ I+C (group A, n=59) } & \multicolumn{2}{|c|}{ I (group B, n=75) } & \multicolumn{2}{|c|}{$C$ (group $C, n=57$ ) } \\
\hline & AE & Grade 3 or 4 & $\mathrm{AE}$ & Grade 3 or 4 & AE & Grade 3 or 4 \\
\hline Neutropenia & 30 (50.8\%) & $8(13.6 \%)$ & $0(0.0 \%)$ & $0(0.0 \%)$ & 24 (42.1\%) & $4(7.0 \%)$ \\
\hline Anemia & 24 (40.7\%) & $2(3.4 \%)$ & $0(0.0 \%)$ & $0(0.0 \%)$ & 29 (50.9\%) & $5(8.8 \%)$ \\
\hline Thrombocytopenia & II (I8.6\%) & $2(3.4 \%)$ & $0(0.0 \%)$ & $0(0.0 \%)$ & $13(22.8 \%)$ & $0(0.0 \%)$ \\
\hline Leucopenia & $13(22.0 \%)$ & $0(0.0 \%)$ & $4(5.3 \%)$ & $0(0.0 \%)$ & 13 (22.8\%) & $3(5.3 \%)$ \\
\hline Rash & $31(52.5 \%)$ & $5(8.5 \%)$ & $23(30.7 \%)$ & $5(6.7 \%)$ & $8(14.0 \%)$ & $0(0.0 \%)$ \\
\hline Stomatitis & $13(22.0 \%)$ & $2(3.4 \%)$ & 7 (9.3\%) & $2(2.7 \%)$ & $3(5.3 \%)$ & $0(0.0 \%)$ \\
\hline Nausea & 23 (39.0\%) & $2(3.4 \%)$ & $4(5.3 \%)$ & $0(0.0 \%)$ & 23 (40.4\%) & $4(7.0 \%)$ \\
\hline Vomiting & $23(39.0 \%)$ & $0(0.0 \%)$ & $5(6.7 \%)$ & $0(0.0 \%)$ & $23(40.4 \%)$ & $2(3.5 \%)$ \\
\hline Diarrhea & $20(33.9 \%)$ & $0(0.0 \%)$ & $16(21.3 \%)$ & $0(0.0 \%)$ & $12(21.0 \%)$ & $2(3.5 \%)$ \\
\hline Constipation & 7 (11.9\%) & $0(0.0 \%)$ & 7 (9.3\%) & $0(0.0 \%)$ & $12(21.0 \%)$ & $0(0.0 \%)$ \\
\hline AST/ALT elevation & $22(37.3 \%)$ & $5(8.5 \%)$ & $9(12.0 \%)$ & $0(0.0 \%)$ & $14(24.6 \%)$ & $2(3.5 \%)$ \\
\hline Fatigue & 15 (25.4\%) & $0(0.0 \%)$ & $5(6.7 \%)$ & $0(0.0 \%)$ & 14 (24.6\%) & $0(0.0 \%)$ \\
\hline Alopecia & $10(16.9 \%)$ & $0(0.0 \%)$ & $0(0.0 \%)$ & $0(0.0 \%)$ & 13 (22.8\%) & $0(0.0 \%)$ \\
\hline Pneumonitis & 3 (5.1\%) & $0(0.0 \%)$ & $0(0.0 \%)$ & $0(0.0 \%)$ & 2 (3.5\%) & $0(0.0 \%)$ \\
\hline
\end{tabular}

Abbreviations: AE, adverse events; ALT, alanine transaminase; AST, aspartate transaminase; C, chemotherapy; I, icotinib; I+C, icotinib+chemotherapy.

and gastrointestinal events, most of which were mild. Grade 3 or 4 adverse events occurred more often with combination therapy and chemotherapy alone. No drug-related interstitial lung disease or drug-related death was observed.

\section{The curative effect of different treatments on exon 19 deletion in the EGFR gene}

\section{Patient characteristics}

A total of 102 patients had EGFR exon 19 deletion. Of these, 31 patients received icotinib+chemotherapy (group A1), 42 patients received icotinib alone (group B1), and 29 patients received chemotherapy alone (group $\mathrm{C} 1$ ). Patients' baseline characteristics (age, sex, smoking history, and ECOG PS) are summarized in Table 4.

\section{Short-term curative effects}

These results are shown in Table 5. The ORR in group A1 was higher than that in group B1 $(74.2 \%$ vs $50 \%, p<0.05)$, while the ORR in group B1 was superior to that in group C1 ( $50 \%$ vs $24.1 \%, p<0.05$ ). The DCR difference between group A1 and group B1 did not reach statistical significance ( $96.8 \%$ vs $92.9 \%, p>0.05)$; the DCR in group B1 was better than that in group $\mathrm{C} 1(92.9 \%$ vs $69 \%, p<0.05)$.

\section{Survival analysis}

The mPFS for the patients in group A1, group B1, and group C1 was 13.391 months (95\% CI, 11.636-15.146), 9.202 months (95\% CI, 8.122-10.283), and 5.655 months (95\% CI, 4.395-6.915), respectively. The patients treated with icotinib+chemotherapy had longer PFS compared to those treated with icotinib alone. In addition, patients treated with icotinib alone had significantly longer PFS compared to those treated with chemotherapy ( $p<0.001$, Figure $2 \mathrm{~A})$. The median OS was 26.096 months (95\% CI, 21.533-30.660) in group A1, 20.470 months (95\% CI, 18.018-22.923) in group B1, and 20.618 months (95\% CI, 16.987-24.248) in group C1. Better OS was observed in group A1 compared to

Table 4 Characteristics of patients with EGFR 19 exon deletion

\begin{tabular}{|c|c|c|c|c|}
\hline Characteristics & I+C (group AI, n=3I) & I (group B I, n=42) & $C($ group $C I, n=29)$ & Total $(n=102)$ \\
\hline Median age (years) & 62.1 & 61.7 & 59.7 & 61.2 \\
\hline \multicolumn{5}{|l|}{ Sex } \\
\hline Male & $35.5 \%(n=I I)$ & $38.1 \%(n=16)$ & $37.9 \%(n=\mid I)$ & $37.3 \%(n=38)$ \\
\hline Female & $64.5 \%(n=20)$ & $61.9 \%(n=26)$ & $62.1 \%(n=18)$ & $62.7 \%(n=64)$ \\
\hline \multicolumn{5}{|l|}{ Smoking history (\%) } \\
\hline Nonsmoker & $58.1 \%(n=18)$ & $66.7 \%(n=28)$ & $65.5 \%(n=19)$ & $63.7 \%(n=65)$ \\
\hline Smoker & $41.9 \%(n=13)$ & $33.3 \%(n=14)$ & $34.5 \%(n=10)$ & $36.3 \%(n=37)$ \\
\hline \multicolumn{5}{|c|}{ ECOG performance status score } \\
\hline $0-1$ & $100 \%(n=3 \mid)$ & $95.2 \%(n=40)$ & $100 \%(n=29)$ & $93.2 \%$ \\
\hline 2 & $0 \%(n=0)$ & $4.8 \%(n=2)$ & $0 \%(n=0)$ & $6.8 \%$ \\
\hline
\end{tabular}

Abbreviations: C, chemotherapy; ECOG, Eastern Cooperative Oncology Group; EGFR, epidermal growth factor receptor; I, icotinib; I+C, icotinib+chemotherapy. 
Table 5 Short-term curative effects of patients with EGFR 19 exon deletion

\begin{tabular}{|c|c|c|c|}
\hline $\begin{array}{l}\text { Curative } \\
\text { effects }\end{array}$ & $\begin{array}{l}I+C(\text { group AI, } \\
n=3 I)\end{array}$ & $\begin{array}{l}\text { I (group B I, } \\
n=42)\end{array}$ & $\begin{array}{l}C \text { (group CI, } \\
n=29)\end{array}$ \\
\hline \multicolumn{4}{|c|}{ Best response } \\
\hline CR & $3.2 \%(n=1)$ & $0 \%(n=0)$ & $0 \%(n=0)$ \\
\hline PR & $71.0 \%(n=22)$ & $50.0 \%(n=2 I)$ & $24.1 \%(n=7)$ \\
\hline SD & $22.6 \%(n=7)$ & $42.9 \%(n=18)$ & $44.8 \%(n=13)$ \\
\hline PD & $3.2 \%(n=l)$ & $7.1 \%(n=3)$ & $31.0 \%(n=9)$ \\
\hline ORR (\%) & 74.2 & 50.0 & 24.1 \\
\hline DCR (\%) & 96.8 & 92.9 & 69.0 \\
\hline
\end{tabular}

Abbreviations: $C$, chemotherapy; $C R$, complete remission; DCR, disease control rate; EGFR, epidermal growth factor receptor; I, icotinib; I+C, icotinib+chemotherapy; ORR, overall response rate; PD, progression disease; PR, partial disease; $\mathrm{SD}$, stable disease.

group B1 ( $p<0.05$, Figure 2B). The OS comparison between group $\mathrm{B}$ and group $\mathrm{C}$ did not reach statistical significance $(p>0.05$, Figure 2B).

\section{The curative effect of different treatments on patients with L858R mutation in the EGFR gene}

\section{Patient characteristics}

A total of 89 patients harbored the L858R point mutation in the EGFR gene. Of these patients, 28 received icotinib+chemotherapy (group A2), 33 received icotinib alone (group B2), and 28 received chemotherapy alone (group C2). The patients' baseline characteristics (age, sex, smoking history, ECOG PS, and EGFR mutation) are summarized in Table 6.

\section{Short-term curative effects}

These results are shown in Table 7. No statistically significant difference was found in the ORR between group A2

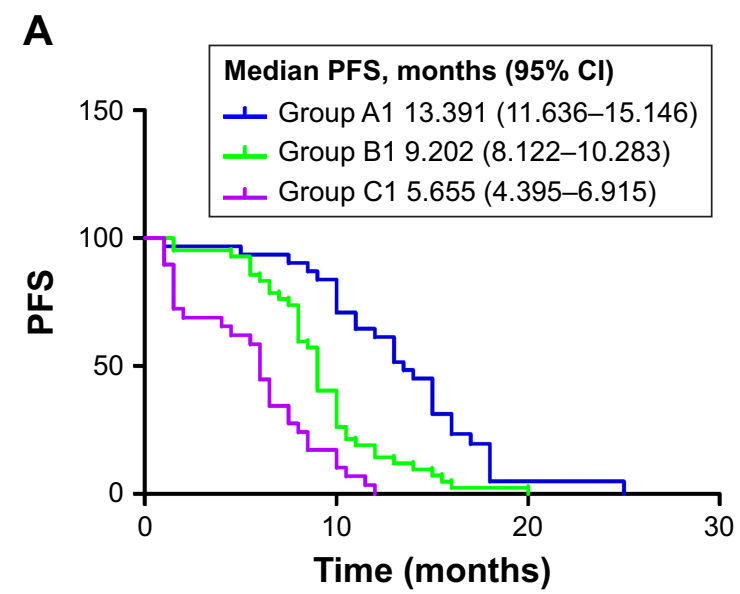

and group B2 (53.6\% vs 42.4\%, $p>0.05)$. While the ORR in group B2 was higher than that in group $\mathrm{C} 2$, there was no statistically significant difference between the two groups (42.4\% vs $28.6 \%, p>0.05)$. The difference in DCR between group A2, group B2, and group C2 did not reach statistical significance ( $92.9 \%$ vs $90.9 \%$ vs $71.4 \%, p>0.05)$.

\section{Survival analysis}

The median PFS for the patients in group A2, group B2, and group C2 was 9.571 months (95\% CI, 8.290-10.853), 7.848 months (95\% CI, 6.692-9.005), and 7.464 months (95\% CI, 6.219-8.709), respectively. The median OS was 21.587 months (95\% CI, 18.251-24.922) in group A2, 19.822 months (95\% CI, 17.343-22.302) in group B2, and 20.125 months (95\% CI, 17.847-22.404) in group C2.

There was a favorable trend in PFS for the patients treated with icotinib+chemotherapy compared to those treated with icotinib alone $(p<0.05$, Figure $3 \mathrm{~A})$, while there was no difference between the PFS in group B2 and group C2 $(p>0.05$, Figure $3 \mathrm{~A})$. Although the OS in group A2 was $\sim 3$ months longer than that in group B2, there was no statistically significant difference between two groups ( $p>0.05$, Figure $3 \mathrm{~B})$. The OS comparison between group B2 and group C2 did not reach statistical significance ( $p>0.05$, Figure $3 \mathrm{~B})$.

\section{Discussion}

Based on large clinical trials, the first-line treatment of choice for advanced NSCLC harboring EGFR mutations is EGFR-TKI. ${ }^{24}$ However, drug resistance seriously reduces the curative effect of TKI. Clinical trials of EGFR-TKI combined with chemotherapy compared to EGFR-TKI alone are becoming more popular in an effort to delay the development of resistance and improve distant disease control. However,

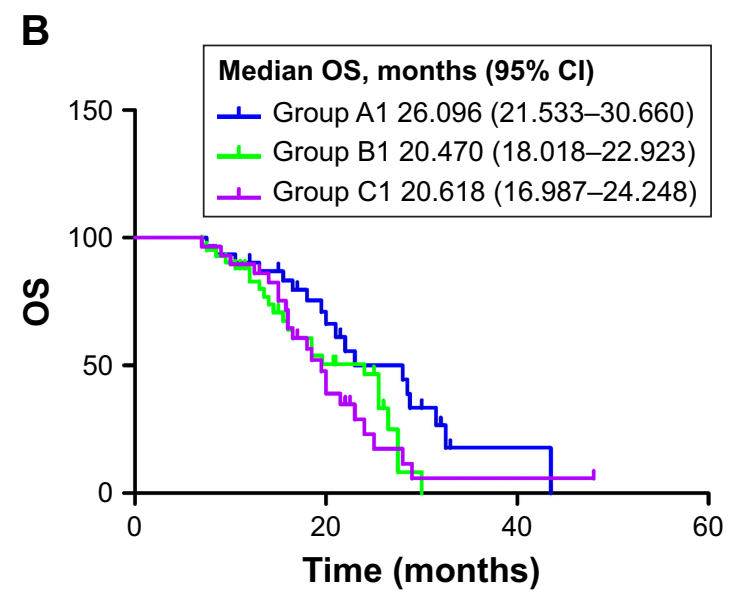

Figure 2 Kaplan-Meier curve of PFS (A) and OS (B) for patients with epidermal growth factor receptor exon 19 deletion.

Notes: Group AI, 3 I patients received icotinib+chemotherapy; Group BI, 42 patients received icotinib alone; Group Cl, 29 patients received chemotherapy alone. Abbreviations: OS, overall survival; PFS, progression-free survival. 
Table 6 Characteristics of patients with EGFR L858R mutation

\begin{tabular}{|c|c|c|c|c|}
\hline Characteristics & I+C (group A2, n=28) & I (group B2, n=33) & $C($ group $C 2, n=28)$ & Total $(n=89)$ \\
\hline Median age (years) & 61.8 & 59.3 & 60.2 & 60.4 \\
\hline \multicolumn{5}{|l|}{ Sex } \\
\hline Male & $46.4 \%(n=13)$ & $36.4 \%(n=12)$ & $57.1 \%(n=16)$ & $46.1 \%(n=4 I)$ \\
\hline Female & $53.6 \%(n=15)$ & $63.6 \%(n=21)$ & $42.9 \%(n=12)$ & $53.9 \%(n=48)$ \\
\hline \multicolumn{5}{|l|}{ Smoking history (\%) } \\
\hline Nonsmoker & $64.3 \%(n=18)$ & $63.6 \%(n=21)$ & $53.6 \%(n=15)$ & $60.7 \%(n=54)$ \\
\hline Smoker & $35.7 \%(n=10)$ & $36.4 \%(n=12)$ & $46.4 \%(n=13)$ & $39.3 \%(n=35)$ \\
\hline \multicolumn{5}{|c|}{ ECOG performance status score } \\
\hline $0-1$ & $100 \%(n=28)$ & $97.0 \%(n=32)$ & $96.4 \%(n=27)$ & $97.8 \%$ \\
\hline 2 & $0 \%(n=0)$ & $3.0 \%(n=1)$ & $3.6 \%(n=1)$ & $2.2 \%$ \\
\hline
\end{tabular}

Abbreviations: C, chemotherapy; ECOG, Eastern Cooperative Oncology Group; EGFR, epidermal growth factor receptor; I, icotinib; I+C, icotinib+chemotherapy.

to date, no consensus has been reached regarding the efficacy of EGFR-TKI combined with chemotherapy in the first-line treatment of patients with NSCLC harboring EGFR mutations. We conducted this retrospective analysis to investigate the overall efficacy of icotinib with or without chemotherapy as first-line treatment for NSCLC patients with EGFR mutations. To our knowledge, ours is the first case-control study comparing the effectiveness of icotinib+chemotherapy and icotinib or chemotherapy alone in EGFR-mutant NSCLC patients.

A total of 191 patients were enrolled in our study; five matching variables (age, sex, smoking history, ECOG PS, and type of EGFR mutation) were selected. Statistically, it is complicated to adjust various types of chemotherapy and use periods for each chemotherapeutic agent. Therefore, the primary end point of this study was PFS rather than OS. For all patients, we found a statistically significant difference in PFS between the three groups, suggesting that the effectiveness of icotinib+chemotherapy was better than that of icotinib or chemotherapy alone and that the effectiveness of icotinib alone was better than that of chemotherapy alone. The results of our study also suggest that icotinib combined with chemotherapy as first-line treatment is associated with a better ORR in advanced NSCLC with EGFR mutations.
Although the OS data are immature, better OS was observed in the combination group compared with the icotinib alone or chemotherapy alone groups, while there was no significant difference between the patients treated with icotinib and chemotherapy, which may be associated with the follow-up treatment. Given the retrospective nature of this study, toxicity profiles were not always complete. We observed that adverse events were more frequent in the icotinib+chemotherapy and chemotherapy groups. Although some patients experienced a grade 3 or higher adverse event in the combination group, these events were predictable and manageable. No occurrence of an interstitial lung disease event was noted in this cohort. All of the drugs were well tolerated, and no treatment-related mortality was observed.

We also observed the curative effect of different treatments on patients harboring different EGFR mutations. The results showed that icotinib combined with chemotherapy significantly prolonged the PFS ( $\sim 4$ months) compared to icotinib or chemotherapy alone for the patients who had the EGFR exon 19 deletion. The combination treatment also significantly prolonged the OS ( $\sim 6$ months) in this group of patients. However, for the patients with the EGFR L858R mutation, although the combination of icotinib and chemotherapy extended the PFS, it did not significantly prolong

Table 7 Short-term curative effects of patients with EGFR L858R mutation

\begin{tabular}{llll}
\hline Curative effects & $\begin{array}{l}\text { I+C } \\
\text { (group A2, } \mathbf{n = 2 8 )}\end{array}$ & $\begin{array}{l}\text { I (group B2, } \\
\mathbf{n = 3 3 )}\end{array}$ & $\begin{array}{l}\text { C (group C2, } \\
\mathbf{n = 2 8 )}\end{array}$ \\
\hline Best response & & & $0 \%(\mathrm{n}=0)$ \\
CR & $0 \%(\mathrm{n}=0)$ & $0 \%(\mathrm{n}=0)$ & $28.6 \%(\mathrm{n}=8)$ \\
PR & $53.6 \%(\mathrm{n}=15)$ & $42.4 \%(\mathrm{n}=14)$ & $42.8 \%(\mathrm{n}=12)$ \\
SD & $39.3 \%(\mathrm{n}=11)$ & $48.5 \%(\mathrm{n}=16)$ & $28.6 \%(\mathrm{n}=8)$ \\
PD & $7.1 \%(\mathrm{n}=2)$ & $9.1 \%(\mathrm{n}=3)$ & 28.6 \\
ORR $(\%)$ & 53.6 & 42.4 & 71.4 \\
DCR $(\%)$ & 92.9 & 90.9 & \\
\hline
\end{tabular}

Abbreviations: C, chemotherapy; CR, complete remission; DCR, disease control rate; EGFR, epidermal growth factor receptor; I, icotinib; I+C, icotinib+chemotherapy; ORR, overall response rate; PD, progression disease; PR, partial disease; SD, stable disease. 
A

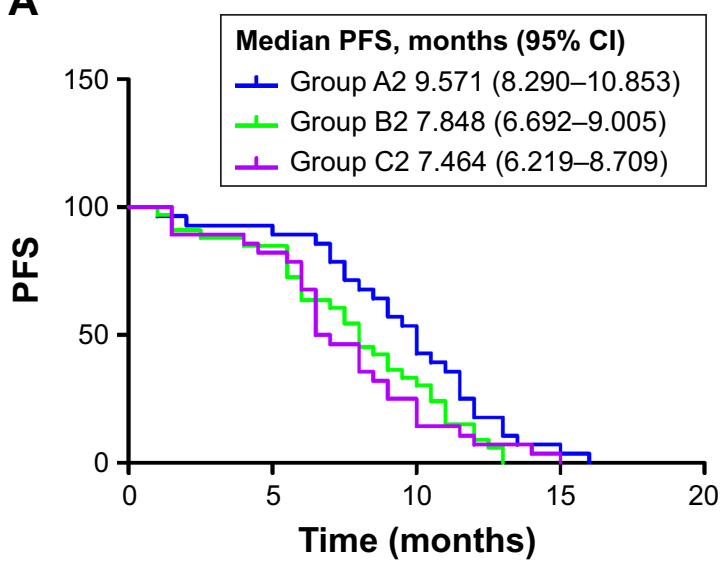

B

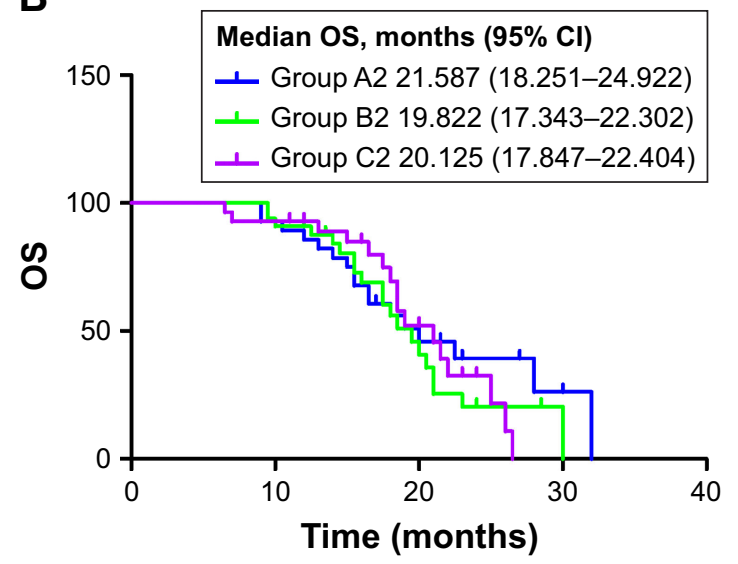

Figure 3 Kaplan-Meier curve of PFS (A) and OS (B) for patients with epidermal growth factor receptor exon 2I L858R mutation.

Notes: Group A2, 28 patients received icotinib+chemotherapy; Group B2, 33 patients received icotinib alone; Group C2, 28 patients received chemotherapy alone. Abbreviations: OS, overall survival; PFS, progression-free survival.

the OS. Previous studies had found that advanced NSCLC patients harboring the EGFR exon 19 deletion may experience a better curative effect when treated with EGFR-TKI compared to the patients who had the L858R mutation. We also found that the patients who had EGFR exon 19 deletion can obtain more survival benefit when treated with icotinib combined with chemotherapy or icotinib alone compared to L858R-mutant patients.

This real-life analysis has several limitations including its small sample size, retrospective nature, and the heterogeneity of treatment regimens. Although five important baseline variables with baseline characteristics were matched between the three groups, the chemotherapy regimens and number of administered treatments varied and were not considered. The difference might have introduced potential bias, which may have affected the outcomes of the study. Furthermore, because of the small number of patients, the analysis is limited in its ability to provide better therapy. Moreover, due to the retrospective nature, other unmeasured confounding factors may have been introduced to the treatment groups. Nevertheless, the study data do reflect clinical practices at that time; we believe that this analysis provides valuable real-life evidence regarding treatments received and outcomes experienced by NSCLC patients with EGFR-sensitive mutations.

\section{Conclusion}

Our results are consistent with previous studies on erlotinib or gefitinib, highlighting that EGFR-mutated advanced NSCLC patients can experience better treatment effectiveness if they are treated with an icotinib/chemotherapy combination compared with icotinib or chemotherapy alone, especially for those harboring the EGFR exon 19 deletion. The modest increase in toxicity was clinically manageable. These results suggest that patients with NSCLC with activating EGFR mutations may obtain a clinical benefit from the addition of chemotherapy to EGFR-TKIs. If the combination of chemotherapy plus EGFR-TKI is used as the first-line treatment, the third-generation EGFR-TKI can then be used after progression if patients are confirmed to have the T790M mutation. ${ }^{12}$ Chemotherapy agents other than the first-line treatment may still be used after progression. The combination of chemotherapy and an EGFR-TKI may be a new treatment option for patients with EGFR mutation-positive NSCLC, which may improve clinical outcomes compared with the current standard of care. Because our study was based on clinical data from a small sample of patients and given the tendency toward longer PFS or OS in patients treated with the icotinib/ chemotherapy combination, larger prospective trials should be conducted to determine the true efficacy and toxicity of these treatments. Indeed, a prospective study with a larger patient population is required to confirm our findings. In addition, the types of chemotherapeutic drugs that can achieve a better curative effect when combined with EGFR-TKI and the possible effects of EGFR-TKI combined with other agents, such as angiogenesis inhibitors or immunocheckpoint inhibitors, are promising avenues for future research.

\section{Acknowledgments}

The authors would like to thank the Chongqing Cancer Hospital (Chongqing, China) for providing the case data of all patients. In addition, the authors would like to thank their mentor and also a co-author for this study, Professor Donglin Wang and Huiwen Ma, from Chongqing Cancer Hospital, Chongqing, China, for their support, incisive comments, and useful suggestions. The present study was supported by the Research Projects of the China cancer fund (grant no LC2016W04). 


\section{Disclosure}

The authors report no conflicts of interest in this work.

\section{References}

1. Ward EM, DeSantis CE, Lin CC, et al. Cancer statistics: breast cancer in situ. CA Cancer J Clin. 2015;65:481-495.

2. Schiller JH, Harrington D, Belani CP, et al; Eastern Cooperative Oncology Group. Comparison of four chemotherapy regimens for advanced non-small-cell lung cancer. $N$ Engl J Med. 2002;346(2):92-98.

3. Amann J, Kalyankrishna S, Massion PP, et al. Aberrant epidermal growth factor receptor signaling and enhanced sensitivity to EGFR inhibitors in lung cancer. Cancer Res. 2005;65(1):226-235.

4. Pao W, Miller V, Zakowski M, et al. EGF receptor gene mutations are common in lung cancers from "never smokers" and are associated with sensitivity of tumors to gefitinib and erlotinib. Proc Natl Acad Sci US A. 2004;101(36):13306-13311.

5. Shigematsu H, Lin L, Takahashi T, et al. Clinical and biological features associated with epidermal growth factor receptor gene mutations in lung cancers. J Natl Cancer Inst. 2005;97(5):339-346.

6. Mitsudomi T, Morita S, Yatabe Y, et al; West Japan Oncology Group. Gefitinib versus cisplatin plus docetaxel in patients with non-smallcell lung cancer harbouring mutations of the epidermal growth factor receptor (WJTOG3405): an open label, randomised phase 3 trial. Lancet Oncol. 2010;11(2):121-128.

7. Zhou $\mathrm{C}, \mathrm{Wu} \mathrm{YL}$, Chen $\mathrm{G}$, et al. Erlotinib versus chemotherapy as first-line treatment for patients with advanced EGFR mutation-positive non-small-cell lung cancer (OPTIMAL, CTONG-0802): a multicentre, open-label, randomised, phase 3 study. Lancet Oncol. 2011;12(8): 735-742.

8. Zwitter M, Rajer M, Stanic K, et al. Intercalated chemotherapy and erlotinib for non-small cell lung cancer (NSCLC) with activating epidermal growth factor receptor (EGFR) mutations. Cancer Biol Ther. 2016;17(8):833-839.

9. Niu FY, Wu YL. Novel agents and strategies for overcoming EGFR TKIs resistance. Exp Hematol Oncol. 2014;3(1):2.

10. Engel J, Richters A, Getlik M, et al. Targeting drug resistance in EGFR with covalent inhibitors: a structure-based design approach. $J$ Med Chem. 2015;58(17):6844-6863.

11. Wang S, Song Y, Yan F, Liu D. Mechanisms of resistance to thirdgeneration EGFR tyrosine kinase inhibitors. Front Med. 2016;10(4): 383-388.

12. Wang S, Cang S, Liu D. Third-generation inhibitors targeting EGFR T790M mutation in advanced non-small cell lung cancer. $J$ Hematol Oncol. 2016;9(1):34.

13. Wang S, Tsui ST, Liu C, Song Y, Liu D. EGFR C797S mutation mediates resistance to third-generation inhibitors in T790M-positive non-small cell lung cancer. J Hematol Oncol. 2016;9:59.
14. Cheng Y, Murakami H, Yang PC, et al. Randomized phase II trial of gefitinib with and without pemetrexed as first-line therapy in patients with advanced nonsquamous non-small-cell lung cancer with activating epidermal growth factor receptor mutations. J Clin Oncol. 2016; 34(27):3258-3266.

15. Herbst RS, Prager D, Hermann R; TRIBUTE Investigator Group. TRIBUTE: a phase III trial of erlotinib hydrochloride (OSI-774) combined with carboplatin and paclitaxel chemotherapy in advanced non-small-cell lung cancer. J Clin Oncol. 2005;23(25):5892-5899.

16. Giaccone G, Herbst RS, Manegold C, et al. Gefitinib in combination with gemcitabine and cisplatin in advanced non-small-cell lung cancer: a phase III trial-INTACT 1. J Clin Oncol. 2004;22(5):777-784.

17. Herbst RS, Giaccone G, Schiller JH, et al. Gefitinib in combination with paclitaxel and carboplatin in advanced non-small-cell lung cancer: a phase III trial-INTACT 2. J Clin Oncol. 2004;22(5):785-794.

18. Gatzemeier U, Pluzanska A, Szczesna A, et al. Phase III study of erlotinib in combination with cisplatin and gemcitabine in advanced non-small-cell lung cancer: the Tarceva Lung Cancer Investigation Trial. J Clin Oncol. 2007;25(12):1545-1552.

19. Hirsch FR, Kabbinavar F, Eisen T, et al. A randomized, phase II, biomarker-selected study comparing erlotinib to erlotinib intercalated with chemotherapy in first-line therapy for advanced non-small-cell lung cancer. J Clin Oncol. 2011;29(26):3567-3573.

20. Jänne PA, Wang X, Socinski MA, et al. Randomized phase II trial of erlotinib alone or with carboplatin and paclitaxel in patients who were never or light former smokers with advanced lung adenocarcinoma: CALGB 30406 trial. J Clin Oncol. 2012;30(17):2063-2069.

21. Cheng Y, Murakami H, Yang PC, et al. Randomized phase II trial of gefitinib with and without pemetrexed as first-line therapy in patients with advanced nonsquamous non-small-cell lung cancer with activating epidermal growth factor receptor mutations. J Clin Oncol. 2016; 34(27):3258-3266.

22. Han B, Jin B, Zhang Y, Chu T, Gu A, Xu J. 1310: combination of chemotherapy and gefitinib as first-line treatment of patients with advanced lung adenocarcinoma and sensitive EGFR mutations: a randomised controlled trial. J Thorac Oncol. 2016;11(4 Suppl):S113-S114.

23. Liang JL, Ren XC, Lin Q. Treating advanced non-small-cell lung cancer in Chinese patients: focus on icotinib. Onco Targets Ther. 2014; 7:761-770.

24. Jackman DM, Yeap BY, Sequist LV, et al. Exon 19 deletion mutations of epidermal growth factor receptor are associated with prolonged survival in non-small cell lung cancer patients treated with gefitinib or erlotinib. Clin Cancer Res. 2006;12:3908-3914.

25. Goto K, Nishio M, Yamamoto N, et al. A prospective, phase II, open-label study (JO22903) of first-line erlotinib in Japanese patients with epidermal growth factor receptor (EGFR) mutation-positive advanced non-small-cell lung cancer (NSCLC). Lung Cancer. 2013;82: $109-114$.
OncoTargets and Therapy

\section{Publish your work in this journal}

OncoTargets and Therapy is an international, peer-reviewed, open access journal focusing on the pathological basis of all cancers, potential targets for therapy and treatment protocols employed to improve the management of cancer patients. The journal also focuses on the impact of management programs and new therapeutic agents and protocols on

\section{Dovepress}

patient perspectives such as quality of life, adherence and satisfaction. The manuscript management system is completely online and includes a very quick and fair peer-review system, which is all easy to use. Visit http://www.dovepress.com/testimonials.php to read real quotes from published authors. 